\title{
Impact of Curative Effect of Laparoscopic and Open Partial Nephrectomy for Complex Renal Tumours on Renal Function
}

\author{
Hong-mei Li1, Peng Sun2, Wen-zeng Yang1 and Zhen-yu Cui1 \\ IDepartment of Urology, Affiliated Hospital of Hebei University, Baoding, Hebei, P. R. China \\ ${ }^{2}$ Department of Cardiovascular Medicine, Affiliated Hospital of Hebei University, Baoding, Hebei, P. R. China
}

\begin{abstract}
Objective: To explore the effect and safety of laparoscopic partial nephrectomy (LPN) and open partial nephrectomy (OPN) in the treatment of complex renal tumours and to compare renal function in the perioperative period.

Study Design: Comparative study.

Place and Duration of Study: Departments of Urology and Cardiovascular Medicine, Affiliated Hospital of Hebei University, from July 2016 to June 2018.

Methodology: A total of 168 complex renal tumour patients with renal tumour scoring system scores (R.E.N.A.L) $\leq 7$ and tumour stages of T1aNOMO TIbNOMO (diameter of tumour $\leq 7 \mathrm{~cm}$ without metastasis) were chosen. The patients were classified into the retroperitoneal laparoscopic partial nephrectomy (RLPN) group and open partial nephrectomy (OPN) group, comprising 84 patients in each group. Intraoperative and postoperative characteristics of both groups were compared. Statistical analysis of the occurrence rate of postoperative complications was conducted. Renal function indicators, including serum urea (UREA), creatinine ( $\mathrm{Cr}$ ), uric acid (UA) and glomerular filtration rate (GFR), were evaluated one day before the operation and one week after the operation.

Results: The differences between the two groups in intraoperative renal warm ischaemia time (WIT) and postoperative drainage time were not statistically significant. Compared with OPN group, RLPN group had shorter recovery time of gastrointestinal function, less use of analgesics and longer hospital stay. The difference in the recent occurrence rate of complications was not statistically significant. The differences between the two groups in UREA, Cr, UA and GFR one day before the operation and one week after the operation were not statistically significant $(p>0.05)$. The $\mathrm{Cr}$ and UA levels of both groups improved significantly after the operation, and the GFR level declined significantly. The differences were statistically significant compared with preoperation levels $(p<0.05)$.

Conclusion: The effect and safety of RLPN in the treatment of complex renal tumours are equal to those of open operations. Laparoscopic surgery involves a small operative wound and contributes to renal function recovery, allowing patients to recover quickly; however, laparoscopic surgery has high technical requirements for its operators.
\end{abstract}

Key Words: Laparoscopic partial nephrectomy, Open partial nephrectomy, Complex renal tumour, Curative effect, Renal function.

How to cite this article: Li HM, Sun S, Yang WZ, Cui ZY. Impact of curative effect of laparoscopic and open partial nephrectomy for complex renal tumours on renal function. J Coll Physicians Surg Pak 2019; 29(10):958-61.

\section{INTRODUCTION}

Renal tumours are common targets in urinary surgery, and $80 \% \sim 90 \%$ of these are malignant. Surgery is the main treatment method of renal tumours. ${ }^{1}$ Complex renal tumours are common clinically. The complexity usually refers to localised tumours of anatomically or functionally isolated kidneys with a tumour scoring system score (R. E. N. A. L.) $\geq 7$ and a tumour stage of T1a Tlb. Nephron-sparing surgery is the main clinical treatment method of complex renal tumours. ${ }^{2}$ For renal tumours with diameters of $\leq 7 \mathrm{~cm}$ and a low stage,

Correspondence to: Zhen-yu Cui, Department of Urology,

Affiliated Hospital of Hebei University, Baoding, Hebei,

071030, P. R. China

E-mail:cvaauy@sina.com

Received: March 26, 2019; Revised: July 02, 2019;

Accepted: July 12, 2019 nephron-sparing surgery (NSS) gradually replaces radical operation to become an important treatment method for renal tumours. ${ }^{3}$ With continuous improvement in laparoscopic surgery, laparoscopic partial nephrectomy (LPN) has become a recommended operation to treat T1 localised renal tumours.4-6 Laparoscopic surgery has the advantages of a small surgical incision, fast postoperative recovery, and small intraoperative blood loss, as well as tumour treatment effectiveness similar to that with open operations.7,8 However, there are high technical requirements for LPN, and the occurrence rate of complications of laparoscopic surgery may be high. ${ }^{9}$ LPN includes two approaches: transabdominal and retroperitoneal laparoscopic partial nephrectomy (RLPN). RLPN is more direct, more rapidly seeks renal pedicles, disturbs visceral organs less, and can avoid peritoneal contamination and organ injury. ${ }^{10}$ 
In this study, the objective was to determine the effect of RLPN and OPN in the treatment of complex renal tumours and on renal function.

\section{METHODOLOGY}

The study was approved by the Institutional Ethics Committee and written informed consents were obtained from all participants. A total of 168 patients who were diagnosed by Urology and Cardiovascular Medicine at Affiliated Hospital of University and received NSS treatment of complex renal tumours from July 2016 to June 2018 were chosen. The patients were classified into RLPN group (84 cases) and OPN group (84 cases), as per random number table. Inclusion criteria were definitively diagnosed through a combination of clinical symptoms, chest X-ray films, abdominal ultrasound/CT/ MRI examinations, R. E. N. A. L. $\geq 7$, tumour stage T1aNOM0 TIbN0M0 (diameter of tumour $\leq 7 \mathrm{~cm}$, without metastasis), no relevant surgical contraindications, and signed informed consent form. Exclusion criteria were severe organic lesions such as those on the heart, liver and lung or other malignant tumours; coagulation function and immune deficiency; comorbidities of hypertension and diabetes; presence of regional lymphatic metastases and distant metastases.

For the RLPN group, patients were placed in a lateral position; after general anaesthesia and cannula insertion, a $3 \mathrm{~cm}$ incision was made $2 \mathrm{~cm}$ upward of and along the midaxillary line and crista iliaca. Blunt dissection of the lumbodorsal fascia was performed, and the balloon was inflated to expand the retroperitoneal gap. The operation incisions were made along the posterior axillary line under the $12^{\text {th }}$ rib and anterior axillary line of the rib margin, and the trocar and laparoscope were placed. Artificial pneumoperitoneum $\left(\mathrm{CO}_{2}\right.$ pressure $\left.15 \mathrm{mmHg}\right)$ was established. Extra peritoneal fat was cleared, and the Gerota fascia was cut open lengthwise. Loose tissue between the renal fatty capsule and renal anaemia capsule was separated. Renal parenchyma and the tumour were then exposed, and the renal pedicle and blood vessels were separated. A bulldog vascular clamp was used to interdict the renal artery. WIT was recorded. The tumour and surrounding renal parenchyma were cut along the tumour edge $0.5 \sim 1 \mathrm{~cm}$. The renal collection system and renal parenchyma wound surface were sutured. A drainage tube was placed at the renal artery, and the operation incisions were closed.

For the OPN group, patients were placed in a lateral position. A diagonal incision was made in the $11^{\text {th }}$ rib or under the $12^{\text {th }}$ rib. The incision was cut open layer by layer to enter the retroperitoneal gap. The other steps are similar to the steps of RLPN.

Intraoperative indicators included intraoperative WIT, operation duration and intraoperative blood loss volume.
Postoperative indicators include the following: postoperative drainage time, postoperative drainage volume, gastrointestinal function recovery time, amount of postoperative analgesic use and total length of hospital stay. Recent complications included bleeding, urine leakage, perirenal haematoma, infection of incisional wound, pleural injury and hypercapnia; renal function indicators including serum urea (UREA), creatinine $(\mathrm{Cr})$, uric acid (UA) and glomerular filtration rate (GFR) were determined one day before the operation and one week after the operation.

SPSS Version 22.0 statistical software was used for data analysis. Enumeration data were expressed as the rate (\%) and tested with the $\chi^{2}$ test. Measurement data were expressed as $\mathrm{x} \pm \mathrm{s}$. Independent sample t-tests were used for inter-group comparisons, and paired sample t-tests were used for intra-group comparisons. $\mathrm{P}<0.05$ indicates that the difference was statistically significant.

\section{RESULTS}

The comparison differences in age, gender, BMI, tumour diameter, tumour position and TNM stage were not statistically significant $(p>0.05)$, as shown in Table I. The difference between the two groups in postoperative drainage time was not statistically significant $(p=1.000)$. The postoperative drainage volume, gastrointestinal function recovery time, amount of postoperative analgesic drug use and length of hospital stay of the RLPN group were significantly lower than those of the OPN group, and the differences were statistically significant $(p<0.001)$.

The differences between the two groups in UREA, $\mathrm{Cr}$, UA and GFR one day before the operation were not statistically significant $(p=0.611,0.598,0.996$, and 0.962, respectively). The $\mathrm{Cr}$ and UA levels of both groups improved significantly after the operation, and the GFR level declined significantly. The differences were statistically significant compared with preoperation $(p<0.001)$ levels. The difference in the UREA levels of the two groups before the operation was not statistically significant $(p>0.611)$. The differences in the two groups in UA and GFR one week after the operation were not statistically significant $(p=0,513$ and $p=0.093$, respectively), as shown in Table II.

\section{DISCUSSION}

With rapid developments in medical imaging and minimally invasive surgery technology, surgical method selection is changing from traditional open operations to minimally invasive surgeries. LPN is expected to replace OPN and become the standard operation to treat renal tumours. In 1993, Winfield reported the first LPN. ${ }^{11}$ In 1994, Gill reported the first RLPN. 12 In 2003, Gill was the first to compare the advantages and disadvantages of LPN and OPN in the treatment of renal tumours, ${ }^{13}$ and 
to discuss the differences between the two operations in terms of WIT, operation duration and intraoperative blood loss. LPN was used to treat renal tumours with a tumour diameter of $4 \sim 7 \mathrm{~cm} .{ }^{2}$ There are still questions about the effectiveness and safety of the two operations, 14 and there is little long-term, large sample and multi-centre data support. These questions were explored in this study.

RLPN is a minimally invasive surgery. With the great progress made in endoscope technology, the indications for operation, such as diameter, position and stage of tumours, can be gradually broadened; in contrast, the operation difficulty progressively increases. The therapeutic effect of RLPN is close to that of OPN. This study found that the intraoperative blood loss, postoperative drainage volume, gastrointestinal function recovery time, amount of postoperative analgesic drug use and length of hospital stay of the RLPN group obviously decreased and shortened compared with that of the OPN group. This finding may be because RLPN offers a clearer surgical view, which helps avoid blood vessel damage. Meanwhile, pneumoperitoneum establishment can reduce the amount of venous bleeding and gastrointestinal tissue traction, with little impact on abdominal wall tissues. In addition, the exposure time of the intestinal canal is short. Thus, RLPN contributes to postoperative intestinal canal function recovery and minimises patient exhaustion. In addition, the wound is small, heals fast and only causes slight pain. Thus, the length of the hospital stay obviously shortens. ${ }^{15}$ Bowlin et al. also considered that RLPN could better promote postoperative recovery. ${ }^{16}$ The length of hospital stay for Chinese patients is notably longer than that reported in foreign literature, which may be because China requires patients who had a partial nephrectomy to be hospitalised until the drainage tube is removed. In contrast, foreign patients will receive rehabilitation treatment in community hospitals. With OPNs, doctors can locate the tumour edge through touch. RLPN operators lack touch feedback and need to carefully examine the tumour. The factors of WIT, operation duration, and postoperative drainage time were almost the same for the two groups. Generally, RLPN is characterised as minimally invasive, with a fast recovery time, but has high technical requirements for its operators. ${ }^{17}$

One challenge of LPN is to shorten intraoperative WIT as much as possible, to ensure that postoperative residual kidneys maintain good renal function and to reduce the risk of postoperative acute and chronic renal failure. The consensus in the field is that a WIT of 25 minutes is the most appropriate inflection point for shortterm or long-term renal function damage. ${ }^{18}$ This study found that if the tumour volume was large, the difficulty for excision and suture techniques will increase. However, the average WIT of both groups was approximately 25 minutes. In addition, the blood flow in the renal artery was completely interrupted, but irreversible kidney damage did not occur. In this study, renal function indicators before and after the operation were measured, and it was found that the $\mathrm{Cr}$ and US levels improved significantly, while GFR levels declined significantly, indicating that the two operations can cause low-range impacts on renal functions. With RLPNs, the time of temporary blood flow interruption to the renal pedicle vessels can be optimised to a certain degree. Meanwhile, pneumoperitoneum establishment can protect renal parenchyma to a certain degree, which makes up for the insufficient blood flow during the vessel blockage time.

Although the two operations have similar indications and effectiveness, RLPN has a certain degree of difficulty because it is conducted through the endoscope. RLPN requires operators to remove the tumour and any suspicious tissue, retain as much normal tissue as possible, and completely suture the renal organs, as thoroughly and as soon as possible. Hence, the operators should have extensive operation experience. Becker et al. statistically analysed the occurrence rate of complications in T1 patients who were treated by the two operations and found that the occurrence rate of

Table I: Statistics of the clinical data $(\mathrm{X} \pm \mathrm{S})$.

\begin{tabular}{|c|c|c|c|c|c|c|c|}
\hline Group & No. & $\begin{array}{c}\text { Gender } \\
\text { (male/female) }\end{array}$ & $\begin{array}{c}\text { Age } \\
\text { (year) }\end{array}$ & $\begin{array}{c}\text { BMl } \\
\left(\mathrm{kg} / \mathrm{m}^{2}\right)\end{array}$ & $\begin{array}{c}\text { Maximum diameter } \\
\text { of tumour }(\mathrm{cm})\end{array}$ & $\begin{array}{c}\text { Tumour stage } \\
\text { (T1a / T1b) }\end{array}$ & $\begin{array}{c}\text { Tumour position } \\
\text { (left /right) }\end{array}$ \\
\hline RLPN group & 84 & $54 / 30$ & $53.9 \pm 8.4$ & $23.1 \pm 4.5$ & $4.0 \pm 1.1$ & $62 / 22$ & $48 / 36$ \\
\hline OPN group & 84 & $60 / 24$ & $54.3 \pm 8.5$ & $23.7 \pm 5.2$ & $4.0 \pm 1.2$ & $58 / 26$ & $56 / 28$ \\
\hline $\mathrm{t} / \chi^{2}$ & & 0.982 & 0.307 & 0.800 & $<0.001$ & 0.467 & 1.615 \\
\hline $\mathrm{P}$ & & 0.322 & 0.759 & 0.425 & 1.000 & 0.495 & 0.204 \\
\hline
\end{tabular}

Table II: Comparison of renal function indicators $(X \pm S)$.

\begin{tabular}{l|c|c|c|c|c|c|c|c}
\hline \multirow{2}{*}{ Group } & \multicolumn{2}{|c|}{ UREA $(\mu \mathrm{mol} \cdot 1-1)$} & \multicolumn{2}{c|}{$\mathrm{Cr}(\mu \mathrm{mol} \cdot 1)$} & \multicolumn{2}{c|}{ UA $(\mu \mathrm{mol} \cdot 1-1)$} & \multicolumn{2}{c}{$\left.\mathrm{GFR}\left[\mathrm{ml} /(\mathrm{min} \cdot 1.73 \mathrm{~m})^{2}\right)\right]$} \\
\cline { 2 - 10 } & Before operation & After operation & Before operation & After operation & Before operation & After operation & Before operation & After operation \\
\hline RLPN group & $4.07 \pm 1.07$ & $4.57 \pm 0.76$ & $124.65 \pm 16.74$ & $174.65 \pm 14.75^{*}$ & $253.39 \pm 32.32$ & $302.70 \pm 38.86^{*}$ & $41.74 \pm 4.17$ & $35.90 \pm 3.99^{*}$ \\
\hline OPN group & $3.99 \pm 0.96$ & $4.11 \pm 0.85$ & $123.42 \pm 13.23$ & $179.61 \pm 13.09^{*}$ & $253.15 \pm 27.90$ & $307.05 \pm 46.83^{*}$ & $41.71 \pm 3.91$ & $36.84 \pm 3.18^{*}$ \\
\hline $\mathrm{t}$ & 0.510 & 3.698 & 0.528 & -2.305 & 0.052 & -0.655 & 0.048 & -1.689 \\
\hline $\mathrm{P}$ & 0.611 & $<0.001$ & 0.598 & 0.022 & 0.9959 & 0.513 & 0.962 & 0.093 \\
\hline
\end{tabular}


complications was almost the same.19 The patients in the laparoscope group recovered more quickly after the operation. Some studies also maintain that while RLPN may reduce trauma and effectively shorten recovery time, it may increase the risk of postoperative complications. 20,21 In this study, the occurrence rate of postoperative complications in the RLPN group was slightly higher than that in the OPN group, but the patients improved after symptomatic treatment.

\section{CONCLUSION}

The effect and safety of RLPN in the treatment of complex renal tumours are equal to those of open operations. Laparoscopic surgery involves a small operative wound and contributes to renal function recovery, allowing patients to recover quickly; however, laparoscopic surgery has high technical requirements for its operators.

\section{ETHICAL APPROVAL:}

The study was approved by the Institutional Ethics Committee of Affiliated Hospital of Hebei University, and ethical approvals were obtained prior to initiation of the research work.

\section{PATIENTS' CONSENT:}

The informed consents were obtained from patients to publish the data concerning this case.

\section{CONFLICT OF INTEREST:}

Authors declared no conflict of interest.

\section{AUTHORS' CONTRIBUTION:}

HML, ZYC: Designed this study and drafted this manuscript. PS: Collected and analysed clinical data.

WZY: Significantly revised this manuscript.

\section{REFERENCES}

1. Zhang WS, Wang LG, Yu H, Guo BB, Han D. Efficacy and safety of retroperitoneal laparoscopic partial nephrectomy in treatment of renal tumors. China J Mod Med 2017; 27:87-90.

2. Liu JM, Chen QK, Xian ZY, Ye CJ, Chen HZ, Feng ZW, et al. The assessment of the safety in retroperitoneal laparoscopic partial nephrectomy for renal tumor. Oncol Prog 2012; 10:285-8.

3. Wang J, Qi L, Zu X, Chen M. Application of retroperitoneal laparoscopic partial nephrectomy for renal cell carcinoma of the early stage. J Cent South Univ 2012; 37:485-91.

4. Ljungberg B, Bensalah K, Canfield S, Dabestani S, Hofmann F, Hora M, et al. EAU guidelines on renal cell carcinoma: 2014 Update. Eur Urol 2015; 67:913-24.

5. Campbell SC, Novick AC, Belldegrun A, Blute ML, Chow GK, Derweesh $\mathrm{IH}$, et al. Guidelines for management of the clinical T1 renal mass. J Urol 2009; 182:1271-9.

6. Liss MA, Wang S, Palazzi K, Jabaji R, Patel N, Lee HJ, et al. Evaluation of national trends in the utilization of partial nephrectomy in relation to the publication of the American
Urologic Association guidelines for the management of clinical T1 renal masses. BMC Urol 2014; 14:101.

7. Gill IS, Kavoussi LR, Lane BR, Blute ML, Babineau D, Colombo JR Jr., et al. Comparison of 1, 800 laparoscopic and open partial nephrectomies for single renal tumors. $J$ Urol 2007; 178:41-6.

8. Domínguez A, Bellido JA, Muñoz-Rodríguez J, AbascalJunquera JM, Hannaoui N, Banús JM. Retroperitoneal and transperitoneal laparoscopic cryotherapy for small renal masses. Actas Urol Esp 2015; 39:582-7.

9. Lane BR, Campbell SC, Gill IS. 10-year oncologic outcomes after laparoscopic and open partial nephrectomy. J Urol 2013; 190:44-9.

10. Xu H, Wang GR, Zhang ZG, Yuan JL, Wang FL. Comparison of therapeutic effects between open and extraperitoneal laparoscopic partial nephrectomy. J Modern Urology 2016; 21:346-8.

11. Winfield HN, Donovan JF, Godet AS, Clayman RV. Laparoscopic partial nephrectomy - initial case report for benign disease. J Endourol 1993; 7:521-6.

12. Gill IS, Delworth MG, Munch LC. Laparoscopic retroperitoneal partial nephrectomy. J Urol 1994; 152(5 pt 1):1539-42.

13. Gill IS, Matin SF, Desai MM, Kaouk JH, Steinberg A, Mascha E, et al. Comparative analysis of laparoscopic versus open partial nephrectomy for renal tumors in 200 patients. J Urol 2003; 170: 64-8.

14. Liu C. Short-term efficacy and safety of laparoscopic partial nephrectomy and partial open nephrectomy for elderly patients with localized renal cell carcinoma. Chinese J Prac Med 2018; 45:74-6.

15. Agrawal V, Sharma A, Wu G. Preoperative fiducial coil placement facilitates robot-assisted laparoscopic excision of retroperitoneal small solitary metastasis of kidney cancer. Urology 2014; 84:21-2.

16. Bowlin PR, Farhat WA. Laparoscopic nephrectomy and partial nephrectomy: Intraperitoneal, retroperitoneal, single site. Urol Clin North Am 2015; 42:31-42.

17. Xu B, Wang JJ, Mi Y, Zhou LQ, Jin J, Zhang Q. Laparoscopic versus open partial nephrectomy for multilocular cystic renal cell carcinoma: A direct comparison based on single-center experience. Urol Int 2015; 94:83-7.

18. Patel AR, Eggener SE. Warm ischemia less than 30 minutes is not necessarily safe during partial nephrectomy every minute matters. Urol Oncol 2011; 29:826-8.

19. Becker A, Pradel L, Kluth L, Schmid M, Eichelberg C, Ahyai S, et al. Laparoscopic ver-SOS open partial nephrectomy for clinical T1 renal masses no impact of surgical approach on perioperative complications and long-term postoperative quality of life. World J Urol 2015; 33:421-6.

20. Marszalek M, Meixl H, Polajnar M, Rauchenwald M, Jeschke K, Madersbacher S. Laparoscopic and open partial nephrectomy: a matched-pair comparison of 200 patients. Eur Urol 2009; 55:1171-78.

21. Springer C, Hoda MR, Fajkovic H, Pini G, Mohammed N, Fornara $\mathrm{P}$, et al. Laparoscopic vs open partial nephrectomy for T1 renal tumours: Evaluation of long-term oncological and functional outcomes in 340 patients. BJU Int 2013; 111: 281-8. 